\title{
Learning Concepts: A Learning-Theoretic Solution to
}

\author{
the Complex-First Paradox.* ${ }^{*}$
}

\author{
Nina L. Poth and Peter Broessel ${ }^{\ddagger}$
}

\begin{abstract}
Children acquire complex concepts like DOG earlier than simple concepts like BROWN, even though our best neuroscientific theories suggest that learning the former is harder than learning the latter and, thus, should take more time (Werning). This is the Complex-First Paradox. We present a novel solution to the Complex-First Paradox. Our solution builds on a generalization of Xu and Tenenbaum's (2007) Bayesian model of word learning. By focusing on a rational theory of concept learning, we show that it is easier to infer the meaning of complex concepts than that of simple concepts.
\end{abstract}

\footnotetext{
*To appear in Philosophy of Science, edited by Andrea Woody and Michelle Pham.

${ }^{\dagger}$ Acknowledgements: We are particularly grateful for the valuable comments of two anonymous reviewers. Earlier versions of this paper have been presented at the Salzburg Conference for Young Analytic Philosophy 2015, the German Society for Analytic Philosophy GAP.9 conference and the Conceptual Spaces at Work 2016 Conference. We would like to thank the participants at these events for their helpful feedback. Special thanks to the members of the Emmy-Noether Research Group "From Perception to Belief and Back Again" and the graduate students in the Philosophy Department at the Ruhr-University Bochum, who gave critical feedback on an earlier draft of this paper. In addition, we want to thank Ben Young for proofreading the manuscript. Research on this paper has been generously supported by an Emmy Noether Grant from the German Research Council (DFG), reference number BR 5210/1-1.

\#This work is highly collaborative and both authors contributed equally to this research. Authorship is given in order of reverse seniority.
} 


\section{Introduction}

The Complex-First Paradox, described by Werning (2010) is as follows: substance concepts ${ }^{1}$ like DOG or MILK (typically associated with concrete noun words like 'dog' and 'milk'2) "are semantically more complex and their neural realizations more widely distributed in [the] cortex than those expressed by the other word classes in question" (Werning, 1097), i.e. attributive concepts like BROWN and WARM, which are typically expressed by adjectives or abstract noun words: 'brown(-ness)', 'warm(-th)'. Thus, in the light of our best neuroscientific theories, one would expect that learning substance concepts would be more difficult than learning attributive concepts, and thus to take not only more effort but also more time. Yet, surprisingly, we observe that young concept learners first understand the meanings of concrete noun words, and then subsequently acquire the meanings of adjectives or abstract nouns (Jackson-Maldonado, Thal, Marchman, Bates, and Gutirrez-Clellen; Nelson; Sandhofer and Smith). This finding presents a puzzle for cognitive scientists and philosophers of mind and language: how is it possible that children learn the meanings of concrete nouns like 'dog' earlier than the meanings of adjectives like 'brown' that denote abstract attributes?

In this paper we present a novel philosophical solution to the Complex-First Paradox. Gärdenfors also outlines an idea for a solution to the complex-first paradox based on his theory of covariance detection mechanisms in conceptual spaces. In section 2 we introduce Werning's Complex-First Paradox and clarify what kind of solution we want to provide in this paper. In

\footnotetext{
${ }^{1}$ Following Werning, as well as philosophers such as Millikan, we use the term 'substance concepts' simply to refer to those concepts that represent "(1) stuffs (gold, milk), (2) real kinds (cat, chair), and (3) individuals (Mama, Bill Clinton, the Empire State Building)" (Millikan, 55). No commitment to a specific metaphysics comes with using the term.

${ }^{2}$ When we refer to terms we follow the usual convention and use single quotation marks. When we refer to concepts we use small capitals.
} 
particular, we advocate a change of perspective-instead of focussing on the neuroscientific perspective of concept acquisition, we focus on theories of rational word and concept learning. Thus, the explanation we want to provide is on the computational level instead of the implementation level (Marr), and concentrates on the learning mechanism for learning concepts, instead of the outcomes of this learning mechanism. That is to say, instead of focusing on the implementation (i.e. the neural realization) of concepts, we focus on the workings of the learning mechanism from an algorithmic/computational-level perspective and ignore the implementational level of this learning mechanism. (In this paper, in general we ignore questions concerning the implementation of the underlying learning mechanism.) In section 3, we introduce one specific computational-level theory of rational word learning introduced by $\mathrm{Xu}$ and Tenenbaum (2007,

henceforth 'XTB'). In section 4 we demonstrate how on the basis of XTB's model the paradox can be solved. In section 5 we conclude with a summary and a critical perspective outlining future directions of research.

\section{The Complex-First Paradox and the Different Levels of Ex- planation}

According to Werning (2010), the divergence between the theoretical predictions and children's actual word- and concept-acquisition behavior can be traced back to a set of propositions, which are each individually plausible but, taken together, lead to a paradox in the sense that they are "apparently inconsistent" and, thus, point at "an explanatory deficit in linguistic theory." (Werning, 1096) 
The propositions that lead to the paradox concern concepts, their neural realization, and the time and effort it takes to acquire them.

1. The meanings of concrete nouns are substance concepts.

2. Substance concepts are semantically more complex and their neural realizations more widely distributed in the cortex than those expressed by abstract attributive concepts like GREEN.

3. For a cortically implemented syntax-semantics interface, the more widely distributed a concept's neural realization is, the more effort it takes to establish a link between the concept and some lexical expression thereof.

4. In ontogeny and phylogeny, capabilities demanding more effort, all other things being equal, can be expected to develop and, respectively, evolve later than those demanding less effort.

5. The meanings of concrete nouns, in ontogeny and (probably) phylogeny, are acquired earlier than those of many-perhaps even all—other word classes, and in particular attributive concepts such as GREEN. ${ }^{3}$

Werning discusses each of the above propositions in detail. For each of them he provides ample empirical evidence that supports the respective empirical claim, even though he is, of course, aware that the available empirical evidence alone cannot establish those propositions beyond reasonable doubt. Our problem does not concern the credibility of these propositions, but the

\footnotetext{
${ }^{3}$ Though nouns are commonly learned earlier than many other word classes, the nouns-prior-to-adjectives phenomenon has received particular attention in the literature on word-learning (see, for instance, Gasser and Smith).
} 
perception of them as a paradox. Of course, Werning is also well aware that strictly speaking (1)-(4) do not imply the falsity of (5) (and thus the word 'puzzle' would perhaps have been more appropriate than 'paradox'). However, they lead us, according to Werning, to expect that the complex substance concepts, which we express by concrete nouns, would be acquired later than abstract attributive concepts. Once we acknowledge that these propositions are not inconsistent, we recognize this puzzle as what it is: a challenge. The challenge is to provide an explanation for why, despite propositions (1)-(4), substance concepts are learned earlier than attributive concepts.

To answer that challenge, we propose adopting a new perspective in two respects. First, Marr (1982) distinguishes three levels of analysis in cognitive science: the computational, the algorithmic/representational, and the implementational. On the computational level we describe what function a cognitive mechanism computes (i.e. the input-output function characterizing the mechanism). On the algorithmic level we describe how exactly this input-output function is computed, i.e. which representations are involved and how these representations are processed by the mechanism. Finally, on the implementational level we describe how the cognitive mechanism and its processes are physically realized. As one can see from the premises, Werning's paradox is obtained by focussing on the level of implementation of our conceptual capacities. Our proposal is to focus on the computational level and to study the input-output function that characterizes the learning mechanism.

Secondly and more importantly, Werning ignores the mechanism that enables us to learn words and concepts. In particular, we are still missing a computational-, algorithmic- or implementationallevel account of how the brain and the mind learns concepts. Werning concentrates instead on the effort and time it takes to physically implement the neural realizations corresponding to in- 
dividual concepts. Thus, he concentrates on the implementation of the outputs of the learning mechanism, but he does not discuss the implementation of the learning mechanism. He argues that, in light of all we know about the implementation of our concepts, establishing the neural realizations of substance concepts should take more time and effort in comparison to the establishment of the neural realizations of abstract attributive concepts. Werning ignores the time and effort it takes to learn these words and concepts and he ignores the learning mechanism. We propose to study exactly this. However-and this follows from our earlier constraint-we will also discuss the learning mechanism from the computational level perspective.

Our twofold shift of focus comes at a price: our approach will be silent on which kind of concept is learned first in phylogeny. To determine which kind of concept is learned first in phylogeny, we would need to study the phylogeny of the human sensory system and the phylogeny of the learning mechanism for learning concepts. But this is not what we are doing. We want to meet the challenge of the complex-first paradox by focusing on the computational aspects of the mechanism of concept learning as we currently find it in infants.

To sum up, we seek to resolve the Complex-First Paradox, not by arguing directly against one of the five propositions, but by providing a computational-level account of concept learning. This novel perspective will allow us to discover a more promising alternative, avoiding the ComplexFirst Paradox by explaining why children learn substance concepts faster than abstract attributive concepts. 


\section{Concept Learning as Bayesian Inference}

\subsection{Xu and Tenenebaum's Account of Concept Learning}

It is helpful to understand word and concept learning as an induction task. The child receives evidence of how a specific word or label is used to refer to certain objects that it can visually perceive. ${ }^{4}$ On this evidential basis the child has to form a hypothesis about what concept is associated with that word or label and on the basis of such a hypothesis children can then categorize further objects as falling under that label. On the picture we propose, concept learning goes hand in hand with word learning. Both word and concept learning are crucial steps to linguistic communication, and both are completed if and only if an appropriate concept has been linked with the given label. More specifically, an agent has linked an appropriate concept $C$ with a label $L$ (as used by the majority of English speakers) if and only if based on $C$ the agent is able to solve the corresponding categorization task: to correctly subsume a new object under the label $L$ iff the majority of English speakers also categorizes the object under $L .^{5}$

Before introducing the framework in a more formally rigorous way, let us introduce the key idea

\footnotetext{
${ }^{4}$ In this paper we concentrate exclusively on visual perception. We want to study the categorization of external world objects on the basis of perceiving these objects and this is best understood for visual perception. In particular, whether the objects of auditory and olfactory perception are external world objects (instead of only their smell and their sound) is often disputed in the literature (O'Callaghan). We do not want to burden the present investigation with philosophical and psychological discussions about the objects of olfactory and auditory perception. Nevertheless we believe that a similar story can be told for learning concepts with the help of all forms of perception. To tell this story we would need to introduce our theory of the content of perceptual experiences first, and due to limitations of space we cannot do this in the present paper. For an outline of our theory of the content of perceptual experiences see Brössel.

${ }^{5}$ Our assumption that one has learned and thus possesses a concept if and only if one is able to solve corresponding categorization task, is not undisputed in philosophy. On the one hand it is inadequate for more abstract concepts like 'war' and 'love'. For this reason we restrict our account of concept learning to perceptual concepts. In this respect, we also assume that children first have to learn the perceptual concept of a dog before they can modify it to also contain conceptual truths about the ancestry and diet of dogs.
} 
at hand with an illustrative example taken from (Xu and Tenenbaum). Suppose a father wants to teach his child the concept of DALMATIAN. He does so by pointing at instances of Dalmatians while uttering the label 'fuxur'. This is the evidence the child receives. Then in a second step the child has to infer a hypothesis about what concept is linked with the label 'fuxur' on the basis of this evidence. Especially since the child only receives positive evidence, i.e. positive instances for the given label, there are many hypotheses that are consistent with that evidence. The label 'fuxur' might mean Dalmatian, Dog, Mammal, Black-White Dotted, etc. The child's task is now to determine the most likely or plausible of these hypotheses, and via trial and error (with the help of corrections by the father) the child will learn that the label 'fuxur' is paired with the concept DALMATIAN.

More formally, we can frame the induction task that children are confronted with as follows: ${ }^{6}$

1. Evidence: A single piece of evidence for a child consists of an observed object $o$ and the (typically uttered) label $L$, with which it has been tagged $(T)$. The complete available evidence concerning some label $L$ then consists of an ordered sequence of so-far observed object-label pairs:

$$
\begin{gathered}
e_{i}^{L}=T\left(o_{i}, L\right) \\
E_{n}^{L}=\left(e_{1}^{L} \wedge \ldots \wedge e_{n}^{L}\right)
\end{gathered}
$$

2. Hypotheses: The set of hypotheses $\mathcal{H}_{L}$ amongst which the child has to choose. The hypotheses have the form $H_{\left\langle C_{i}, L\right\rangle}$ and state that some specific concept $C_{i}$ is paired with

\footnotetext{
${ }^{6}$ For simplicity we assume the concept learner acquires evidence with respect to only one label.
} 
the label $L:{ }^{7}$

$$
\mathcal{H}_{L}=\left\{H_{\left\langle C_{i}, L\right\rangle} \mid \text { where } C_{i} \text { is a concept. }\right\}
$$

As is standard in Bayesian cognitive science, $\mathrm{Xu}$ and Tenenbaum treat the relevant learning mechanism (on the computational level) as a Bayesian learning mechanism. On XTB's account, children learn the meaning of labels, i.e. concepts, as if they were Bayesian agents, and thus as if they were using Bayes's Theorem to choose the winning hypothesis.

Theorem 1. Bayes's Theorem in the Context of Concept Learning

$$
\operatorname{Pr}\left(H_{\left\langle C_{i}, L\right\rangle} \mid E_{n}^{L}\right)=\frac{\operatorname{Pr}\left(E_{n}^{L} \mid H_{\left\langle C_{i}, L\right\rangle}\right) \operatorname{Pr}\left(H_{\left\langle C_{i}, L\right\rangle}\right)}{\sum_{C_{j}} \operatorname{Pr}\left(E_{n}^{L} \mid H_{\left\langle C_{j}, L\right\rangle}\right) \operatorname{Pr}\left(H_{\left\langle C_{j}, L\right\rangle}\right)} .
$$

As a result the most probable hypothesis $H_{\left\langle C_{i}, L\right\rangle}$ will be the one that exhibits a good mixture between a high prior probability, $\operatorname{Pr}\left(H_{\left\langle C_{i}, L\right\rangle}\right)$, and a high likelihood of the evidence given the hypothesis, $\operatorname{Pr}\left(E_{n}^{L} \mid H_{\left\langle C_{i}, L\right\rangle}\right)$. How, though, can these probabilities be determined for each of the hypotheses in $\mathcal{H}_{L}$ ?

XTB concentrate on providing an account of how the likelihoods are to be determined in the given setting. For determining $\operatorname{Pr}\left(E_{n}^{L} \mid H_{\left\langle C_{i}, L\right\rangle}\right)$ we need to explain how likely the observational evidence of $n$ objects falling under the label $L$ is, under the assumption that the label $L$ is

\footnotetext{
${ }^{7}$ Nothing here commits us to the view that the hypothesis space and concepts available to an agent are innate. This is a computational-level account of word and concept learning, and we only model the task of concept learning and the solutions to which the children come. In this paper we remain silent on how children solve the task, i.e. on how exactly the brain calculates the computational function we are describing and how it is implemented. Colombo is one of the best papers available on the question of what role Bayesian modelling can play for the empiricismnativism debate.
} 
associated with the concept $C_{i}$. Accounting for the likelihoods is the key step in explaining word and concept learning in general and resolving the Complex-First Paradox in particular. The likelihoods encode how children learn words and concepts; after all, the priors are fixed before any learning takes place and they are not revised in light of the incoming evidence. Only the likelihoods change when the evidence comes in. In order to understand the basic idea of their proposal, let us return to the above example of how the child learns that the concept DALmatian is to be linked with the label 'fuxur'. Suppose a child has the following evidence, $E_{4}^{\text {fuxur }}=\bigwedge_{1}^{4} e_{i}^{\text {fuxur }}$, where each of the $e_{i}^{\text {fuxur }}$ has the form $\left\langle o_{i}, f u x u r\right\rangle$ and the observed objects $o_{i}$ are the following:

$$
\begin{aligned}
& o_{1}=\text { a black-on-white spotted Dalmatian } \\
& o_{2}=\text { a black-on-white spotted Dalmatian } \\
& o_{3}=\text { a brown-on-white spotted Dalmatian } \\
& o_{4}=\text { a black-on-white spotted Dalmatian }
\end{aligned}
$$

Such evidence already excludes many hypotheses in the hypothesis space. In particular, it excludes hypotheses that pair the label 'fuxur' with a concept that does not include Dalmatians, such as CAR, CAT, BLACK, etc. However, there are still many candidate concepts that might be paired with the label, for example the hypotheses:

$$
\begin{aligned}
& H_{\text {Dal }} \text { : 'fuxur' means Dalmatian: 〈Dalmatian, 'fuxur'〉 } \\
& H_{D} \text { : 'fuxur' means Dog: 〈Dog, 'fuxur'〉 } \\
& H_{M} \text { : 'fuxur' means Mammal: 〈MAMmal, 'fuxur'〉 }
\end{aligned}
$$


$H_{A}$ : 'fuxur' means Animal: 〈ANIMAL, 'fuxur'〉

$H_{b}$ : 'fuxur' means BLACK-OR-BROWN-SpOTS-ON-White: 〈BLACK-OR-BROWN-SpOTSON-WHiTE, 'fuxur'>

Intuitively, the child should believe most strongly that 'fuxur' means DALMATIAN (i.e. the hypothesis $\left.H_{D a l}\right)$. This is certainly the hypothesis that most adults would accept upon getting the evidence $E_{4}^{f u x u r}$. Given our assumption that children learn as if they were Bayesians, we have to ask how to set likelihoods in such a way that children can learn that 'fuxur' means DALMATIAN. To answer that question XTB propose what they call the size principle.

The Size Principle: 8

$$
\operatorname{Pr}\left(E_{n}^{L} \mid H_{\left\langle C_{i}, L\right\rangle}\right)=\left(\frac{1}{\operatorname{size}\left(C_{i}\right)}\right)^{n} \text {, if all } n \text { objects labeled } L \text { fall under the concept } C_{i}
$$

and 0 otherwise.

As already explained, the evidence $E_{n}^{L}$ specifies for $n$ objects that they have been labeled $L$. The size principle states that the probability of this observation to be true given that the label $L$ is associated with the concept $C_{i}$ is, if all $n$ objects fall under that concept, proportional to the size of the concept $C_{i}$. As a first pass at understanding the size principle, the size of a concept is

\footnotetext{
${ }^{8}$ We make some changes to the original formulation from XTB. In particular, XTB use the following formulation: $\left(\frac{1}{\text { size }(h)}\right)^{n}$. It seems to us instead that equation (4) is more accurate because hypotheses are indexes for concept-label pairs and thus cannot have a size themselves. It seems correct to us to take the size to be instead about the narrowness of a set of possible objects that is indicated by a concept, and replace size $(h)$ with size $\left(C_{i}\right)$, for the $i t h$ concept. We changed the $n$ term in the denominator because the number of observations cannot be negative.
} 
the number of objects falling under the concept, i.e. the cardinality of the concept's extension. The size principle is supported by the assumption that if the father wants to teach the use of the label $L$ which is associated with the concept $C_{i}$, he draws the $n$ examples randomly from the set of all objects in the concept's extension. For one object, the probability of $E_{1}^{L}$ given that $C_{i}$ is

associated with $L$ is $\frac{1}{\operatorname{size}\left(C_{i}\right)}$.For two objects $o_{1}$ and $o_{2}$ the relevant probability is $\frac{1}{\operatorname{size}\left(C_{i}\right)}$, because the two draws are independent of each other. With the help of this size principle XTB can explain what they term the "suspicious coincidence" effect. To explain this, let us return to our example of the four observed Dalmatians. If the four exemplars are a random sample, it is highly unlikely that we will observe all and only Dalmatians being labeled $L$ if in fact the label is associated with the concept 'dog' or the concept 'black or brown spots on white'. Therefore, as a general rule of inference, given some few and very similar exemplars for a label $L$, it is more plausible to infer that the label is associated with a concept with a smaller (as opposed to larger) extension.

Of course, taken literally this story must be false as children do not have access to the true size of the extension of a given concept. However, as XTB elaborate in their paper, children do have access to a psychological proxy, the similarity between the observed and labeled objects. (Assuming that the size principle is grounded in similarity judgments, which are ultimately grounded in our perceptual system, could also serve as an explanation for why, in concept learning, all or almost all humans learn as if the size principle were true.) Concerning this issue there are certainly more questions that have to be asked and answered. Unfortunately, XTB do not address these questions in their paper, but we ask and answer some of them in Brössel, Brössel and Poth, and Poth. Roughly the idea that we present there is that-following philosophers such as Gärdenfors and psychologists such as Shepard-we assume that perceptual concepts 
correspond to regions in a phenomenal similarity space. On this basis, we spell out a second understanding of XTB's size principle. Instead of identifying the size of a perceptual concept with its extension, we propose to identify the size of a perceptual concept with the size of the corresponding region in such a phenomenal similarity space. In line with Gärdenfors's and Shepard's interpretations, we interpret the size of such a region to be a proxy for how perceptually similar, on average, a concept's instances are. We refer to this definition of 'size' in the current context as the narrowness of a set of possible examples for a concept.

This second understanding of the size principle obviously suggests different predictions concerning concept learning than the first. Concepts such as DICE and FAIR DICE have different extensions but they are perceptually almost indistinguishable. Thus, learning DICE and FAIR DICE from positive examples alone should, according to our preferred understanding of the size principle, result in almost identical learning behaviour. More generally, such a reading of the size principle predicts a similar learning behaviour for both concepts because DICE and FAIR DICE correspond to roughly the same regions in phenomenal similarity space. In contrast, the first understanding would predict a huge difference in learning behaviour since the extensions of fair dice (or precision dice as used in casinos) is much smaller than the extension of dice. Another relevant comparison would be between the concepts INSECT and MAMMAL. The extension of all insects is without doubt much greater than the extension of all mammals. But concerning the perceptual in-between-similarity comparison between insects and mammals this question cannot be answered so clearly. Thus, again we would expect roughly similar learning speeds for the concepts INSECT and MAMMAL if we presupposed the second similarity-based understanding of the size principle, and we would expect vastly different learning speeds if we presupposed the cardinality-based reading of the size principle. The formal details and the philo- 
sophical advantages as well as the empirical predictions and the existing empirical support for our preferred reading of the size principle is discussed in Brössel and Poth. Here we focus on solving the Complex-First Paradox and for this purpose we discuss only examples for which the difference between both understandings of the size principle can be neglected.

\subsection{Empirical Support for Xu and Tenebaum's Account}

To test their Bayesian model, XTB conducted a series of experiments with adults and 3-4 year

old children. Before discussing their results, let us focus on what the theory predicts and return to our worked example.

Intuitively, we would say that the set of Dalmatians is narrower than the set of dogs. In addition, Dalmatians are typically much more similar to each other than dogs are to each other. Thus, whatever understanding of the size principle one presupposes here, for the two alternative hypotheses $H_{D a l}$ and $H_{D}$ the corresponding likelihoods should favor $H_{D a l}$ over $H_{D}$ (if we suppose that we observe one object labeled 'fuxur' that is an instance of both concepts). The likelihoods after 1 observation and after 4 observations look like this.

$$
\begin{aligned}
& \operatorname{Pr}\left(E_{1}^{\text {fuxur }} \mid H_{\text {Dal }}\right)=\left[\frac{1}{\text { size }(\text { Dalmatians })}\right]^{1}>\operatorname{Pr}\left(E_{1}^{\text {fuxur }} \mid H_{D}\right)=\left[\frac{1}{\text { size }(\text { dogs })}\right]^{1} \\
& \operatorname{Pr}\left(E_{4}^{\text {fuxur }} \mid H_{\text {Dal }}\right)=\left[\frac{1}{\text { size }(\text { Dalmatians })}\right]^{4}>\operatorname{Pr}\left(E_{4}^{\text {fuxur }} \mid H_{D}\right)=\left[\frac{1}{\text { size }(\text { dogs })}\right]^{4}
\end{aligned}
$$


Given these likelihoods we can ask what impact the difference in likelihoods has on the difference in conditional probabilities $\operatorname{Pr}\left(H_{D a l} \mid E_{n}^{\text {fuxur }}\right)$ and $\operatorname{Pr}\left(H_{D} \mid E_{n}^{\text {fuxur }}\right)$ if measured by the posterior ratio $\frac{\operatorname{Pr}\left(H_{D a l} \mid E_{n}^{\text {fuxur }}\right)}{\operatorname{Pr}\left(H_{D} \mid E_{n}^{\text {ffuxur }}\right)}$.

$$
\frac{\operatorname{Pr}\left(H_{\text {Dal }} \mid E_{n}^{\text {fuxur }}\right)}{\operatorname{Pr}\left(H_{D} \mid E_{n}^{\text {fuxur }}\right)}=\left[\frac{\operatorname{Pr}\left(E_{1}^{\text {fuxur }} \mid H_{D a l}\right)}{\operatorname{Pr}\left(E_{1}^{\text {fuxur }} \mid H_{D}\right)}\right]^{n} \times \frac{\operatorname{Pr}\left(H_{\text {Dal }}\right)}{\operatorname{Pr}\left(H_{D}\right)}
$$

We know that $\frac{\operatorname{Pr}\left(E_{1}^{\text {fuxur }} \mid H_{D a l}\right)}{\operatorname{Pr}\left(E_{1}^{\text {fixur }} \mid H_{D}\right)}>1$, due to the size principle and the difference in size of the concepts DAlmatian and Dog. Clearly, one instance of the label 'fuxur' might not be enough to lead to an important difference in the conditional probabilities $\operatorname{Pr}\left(H_{\text {Dal }} \mid E_{1}^{f u x u r}\right)$ and $\operatorname{Pr}\left(H_{D} \mid E_{1}^{f u x u r}\right)$. However, obtaining more evidence leads to important differences-the difference between the left and the right side in equation 3 is smaller than the difference between the left and the right side in equation 4. Indeed, the weight of the likelihood ratio $\frac{\operatorname{Pr}\left(E_{n}^{f u x u r} \mid H_{D a l}\right)}{\operatorname{Pr}\left(E_{n}^{\text {fuxur }} \mid H_{D}\right)}$ for determining the posterior ratio $\frac{\operatorname{Pr}\left(H_{D a l} \mid E_{n u r}^{\text {fuxur }}\right)}{\operatorname{Pr}\left(H_{D} \mid E_{n}^{f_{n}}\right)}$ grows exponentially, the more instances of the label 'fuxur' we encounter. Thus, the size of the concept-ignoring for the moment the question of whether the size of the concept is understood in terms of the size of the set of objects falling under the concept or in terms of the phenomenal similarity of the (observed) instances-determines, according to XTB's account, how fast we learn one concept as compared to another concept.

Correspondingly, XTB's account of word and concept learning predicts two general effects that we should observe when we study the word-meaning generalization behaviour of children and adults. First, equation 3 predicts that children and adults will prefer smaller concepts like DAL- 
MATIAN over broader ones like DOG given an equal number of examples. Second, equations 2 and 5 predict that the more compatible examples learners have observed for the alternative concepts, the more strongly they will express their preference for learning smaller concepts like DALMATIAN over larger concepts like DOG. In other words, with additional compatible examples, learners should restrict their generalizations even more towards smaller concepts.

To test these predictions, XTB did a word-learning experiment with a training and a test phase. In the training phase, they presented adults or children with one or three labeled pictures of objects. For instance, they showed one or three pictures of a Dalmatian together with the label 'fuxur'. In the test phase, XTB asked subjects to generalize the label (e.g. 'fuxur') to other objects from an array with a variety of different kinds of objects (e.g. an array with other kinds of animals, vegetables, or vehicles). The subjects' extent of generalization was then used as a measure of their understanding of what the label means.

To test the second prediction, XTB manipulated the number of the examples (e.g. 1 or 3 ) in the training phase. To test the first prediction, XTB manipulated the range spanned by the objects within the 3-example trials. In particular, they presented subjects with either 3 labeled objects from the same subordinate category (e.g. 3 pictures of a Dalmatian), 3 labeled objects from the same basic-level category (e.g. 1 picture apiece of a Dalmatian, a terrier, and a mutt), or 3 labeled objects from the same superordinate category (e.g., 1 picture of each a Dalmatian, a toucan, and a pig).

XTB's results confirm both predictions. First, learners restricted their generalizations of a word towards the smallest consistent category level in the 1-example trials. For instance, given 1 Dalmatian labeled 'fuxur' subjects were more likely to label also other Dalmatians than other 
dogs from the test array with 'fuxur'. Second, learners generalized more gradually in 1-example trials and restricted their generalization behaviour more strongly and distinctively when they had been given 3 compatible examples. For example, when learners saw 1 Dalmatian called 'fuxur', they were less likely to pick out any other dogs or any other non-dog animals in the array. In that case, they were more likely to pick out the other Dalmatians. But given additional pieces of evidence in the training phase (e.g. 2 other Dalmatians called 'fuxur'), learners would typically pick out all and only Dalmatians in the test array, thereby indicating that they believed more strongly that 'fuxur' means DALMATIAN and neither DOG nor ANIMAL.

From the perspective of the size principle, it is to be expected that learners would restrict their generalization behavior to the smallest consistent category level in the test array. Likewise, we would expect that subjects' generalization behavior would become more distinct with multiple examples because, following equation 5 , the evidence in the 1-example cases should obtain a lower weight than in the 3-example cases, and therefore it should be easier for a Bayesian learner to rule out competing alternative hypotheses with three examples than with one example. Thus, taken together, XTB's results are consistent with the predictions from the Bayesian model and thereby illustrate that it is useful to think of word and concept learning as a rational-inductive inference task. The next section establishes that this characterization of word and concept learning is useful for making sense of the complex-first paradox. 


\section{The Explanation of the Complex-First Paradox and Possi- ble Objections}

Let us assume, with XTB, that on the computational-level, word and concept learning can be understood as Bayesian inference and that something like the size principle is in place. Building on these assumptions we can now return to the main goal of this paper: to explain the ComplexFirst Paradox.

Recall that the Complex-First Paradox consists in the fact that children learn complex, substance concepts like DOG or MILK (typically associated with concrete noun words) earlier than simple, attributive concepts like BROWN and WARM (typically expressed by adjectives or abstract noun words). We have to explain why children learn the former type of concept faster than the latter type of concept. From the Bayesian perspective that we are adopting here, since we have not made any assumptions about the priors, we have to explain this with the help of likelihoods. Following XTB, we assume that likelihoods obey the size principle. The less comprehensive a concept is, the higher the likelihood. Thus, to explain the Complex First Paradox, we need only establish one additional claim, i.e. that often complex substance concepts like DOG and MILK are smaller in size than simple, attributive concepts like RED and WARM.

Let us compare the concepts DOG and BROWN as an example. The set of all dogs is much narrower than the set of all brown things. The number of brown insects (which is much smaller than the number of brown things) is already much larger than the number of dogs. Thus, even if we understand size only in terms of the concept's extension, DOG is already much smaller than BROWN INSECT. And we come to the same conclusion when we use the similarity-based 
interpretation of the size of these concepts. Typically, objects that fall under an attribute concept like BROWN are similar to each other in only one respect. They are similar in color but might be extremely dissimilar to each other in all other respects: shape, height, weight, movement, sound, etc. In contrast, objects that fall under substance concepts are often similar to each other in many respects. In the case of basic-level concepts such as DOG, for example, various instances of that concept are similar in shape, color, sound, movement, and behaviour. Often the objects falling under a substance concept are not extremely similar to each other in one specific respect, but considerably similar regarding all respects in which we can perceptually discriminate. We should therefore expect instances of BROWN to be overall less similar to each other than instances of DOG. Thus, also under a similarity-based interpretation DOG has a smaller size than BROWN.

We can thus assume, ceteris paribus, that (subordinate and basic level) substance concepts are smaller than attributive concepts, and these concepts can be learned faster because of the size principle. (It is important to note that one probably cannot establish this for all such concept comparisons. The substance concept METAL is arguably similar in size when compared with the attribute concept METALLIC and the various instances of metal and metallic are probably equally similar. Similarly for superordinate concepts like MAMMAL, we can expect that they display only a very small overall similarity and that they are not much more similar to each other than instances of the attributive concept HAIRY.) With this last assumption at hand, we are now in the position to explain the Complex-First Paradox.

P1 Ceteris paribus, (subordinate and basic level) substance concepts are narrower than attributive concepts. 
P2 Learners will learn narrower concepts earlier than less narrow concepts given comparable numbers of observed instances of the given concepts.

C Learners will learn complex concrete noun concepts earlier than simple attributive concepts, given comparable numbers of observed instances of the given concepts.

The conclusion of this argument shows what we want to show: one can expect that children will learn substance concepts earlier than attributive concepts. The reason is that the former concepts are typically narrower than the latter, which makes them easier to learn for children because of the size principle. This resolves Werning's puzzle.

There are two empirical findings that are seemingly in conflict with our explanation of Werning's complex-first-paradox and the size-principle: the basic-level bias and the shape bias. The basiclevel bias is an inductive preference for basic-level concepts over superordinate and subordinate concepts. The shape bias is an inductive preference for concepts that have a high variability with respect to attributes like, for example, color but not with respect to shape.

Let us discuss the basic-level bias first. In particular, Rosch and Mervis (e.g. Rosch and Mervis; Rosch) argued that concepts are structured in terms of the family resemblances-correlations amongst shared attributes — of their instances. Instances of narrower concepts are more similar because they resemble each other in terms of more attributes than instances of broader concepts, which are less specific. Rosch and Mervis use their structural account to justify the taxonomy of concepts with different sizes on which we rely here. Narrower concepts are positioned at the subordinate level, which is the least inclusive level. Broader concepts combining the least similar instances are positioned at the superordinate level, which is the most inclusive level. Basic-level concepts are in between. The problem for our explanation of the complex-first paradox is that a 
number of studies show that preschoolers and adults prefer to generalize concepts on the basiclevel over the subordinate level (Horton and Markman; Mervis and Crisafi). However, basiclevel concepts are relatively broad and typically span over a range of subordinate concepts. The evidence for the basic-level bias presents a challenge to our account because the size principle would predict evidence for a subordinate-level bias instead. Rosch and Mervis believe that basic-level concepts are preferred because they are the inductively most useful type of concept of the three, since they maximize the similarity amongst instances of a single concept while minimizing their similarity to instances of other concepts.

As already said, the shape bias is a preference for concepts that have a high variability with respect to attributes like color but not with respect to shape. Indeed, amongst the earliest concepts that children learn we find many concepts for artifacts like chair and table, for which color is in fact often irrelevant (Smith et al., 2002). The size principle stands in conflict with the shape bias since it predicts a preference for narrower concepts with respect to both shape and color. If children immediately generalize to concepts for which color is not as relevant as shape, they would obey the size principle only with respect to shape but not with respect to color. This bias has been found in children as young as around 24 months (e.g., Jones, Smith, and Landau; Landau, Smith, and Jones).

What these findings suggest is that children bring a lot of prior knowledge (if we want to call these biases 'knowledge') to the word-learning task. Where do these inductive biases come from? One option is that both of these very different biases are innate. Against this proposal, $\mathrm{Xu}$ and Tenenbaum, 2384 provide evidence that four-year olds, in contrast to adults, do not show a basic-level bias in the Bayesian word-learning paradigm that we have outlined above. If a basic-level bias was innate, one would need to explain why children unlearn it at the age of 
four and re-learn it when they become adults. Based on these results and further evidential data, XTB $(2007,262)$ suggest that the basic-level bias is learned: with more experience, children's empirical prior for "medium-sized" basic-level concepts gets higher and higher because most concepts that parents use and teach in everyday life are medium-sized, basic-level concepts.

Similarly, Smith et al. (2002) provide evidence for the claim that the shape bias is also acquired. They designed a word-learning task to train 17-month-old children with labels for objects that had the same shapes but varied in size, texture, and color. They then tested whether children would generalize the label to novel objects that matched in shape but varied in another attribute. Initially children did not display the bias, but at the end of the training they did. Given that typically children do not show the bias before they are 24 months old, Smith et al.'s results indicate that the shape bias can be developed during experimental training. Based on further evidence, Smith et al. (2006) conclude that the shape bias is acquired and that it develops over time, depending on the perceptual properties of artifacts. Their own attentional learning account explains this development on the basis of "statistical regularities and the higher order generalizations that emerge from them" (ibid., 1339), but they do not provide a model that describes how the shape bias is acquired by young children. Kemp, Perfors, and Tenenbaum provide such a model. They argue that the shape bias is the result of a process of learning to learn concepts. After learning the first couple of concepts, children learn that many concepts allow for a greater variability of colors. Their approach assumes the framework of hierarchical Bayesianism and they show that it is compatible with a wide range of data. If children learn the shape bias, they learn to learn concepts that allow for a variability with respect to color. From this perspective, the shape bias takes the role of an empirically acquired prior and is distinct from the size principle. 
This section showed that one of the most developed and successful (computational-level) theories of concept learning-XTB's Bayesian account—can also explain the complex-first paradox. But there are various empirical phenomena that a successful theory of concept learning needs to be able to explain besides the complex-first paradox. The shape bias and the basic-level bias belong to these phenomena. This section also showed that these biases are compatible with our explanation of the complex-first paradox because they are learned. The complex-first paradox, however, is more basic and asks why the first concepts that children learn, even before they acquire any of the biases, are concrete noun concepts. In this sense, Werning's complex-first paradox is the first of many challenges that a theory of concept learning needs to explain.

\section{Conclusion and Future Perspectives}

Our current best neuroscientific theories of concept learning are at odds with the empirical evidence on word learning, according to Werning (2010). According to our best neuroscientific theories, children should learn concrete noun concepts like DOG later than abstract attributive concepts like BLUE, but children in fact learn such concrete nouns earlier than abstract attributive ones. In this paper, we have presented a novel solution to Werning's (2010) Complex-First Paradox.

Our solution to the paradox starts from Marr's (1982) level of computational theory and specifies concept learning as a computational problem. XTB's (2007) Bayesian model of word learning is a worked example for this idea. In Bayesian word learning, the size principle that is characteristic of the concept-learning mechanism is the input-output function. 
The size principle predicts that, ceteris paribus, children favor smaller over larger concepts because they are associated with greater likelihoods in the word-meaning inferences. In this paper, we have suggested that this rationale also explains why children learn the meanings of concrete nouns earlier than they learn the meanings of abstract attributes. Roughly, concrete noun concepts like DOG are smaller than abstract attributive concepts like BROWN. Thus, concrete noun concepts are easier to infer because the probabilities that they are associated with are greater than the probabilities associated with their abstract attributive alternatives. We have argued that the Bayesian model of word learning can thus be expanded towards a solution to the ComplexFirst Paradox. We conclude that instead of focusing all attention on the neural implementation of concepts, it is important to specify what kind of computational problem concept learning is, and to find appropriate constraints on the function with which it can be solved.

While our solution to the paradox has focused on the computational problem of concept learning, it is worth noting that this cannot be sufficient for a full theory of concept learning. Thus, the next step for a theory of concept learning, aside from the specification of the computational processes in the learning mechanism, is to investigate the representational problem of concept learning and to specify the properties of the mental representations that are used to compute the size of a concept. In addition, one must investigate how specifically to combine such a theory of concept learning with the various acquired biases of concept learning, specifically the shape and the basic-level bias. Future work in this area needs to build in this new foundation of mental representations for concept learning. This new foundation should not only allow for explaining concept learning, but also for explaining learning to learn concepts. 


\section{References}

Werning, Markus. "Complex First? On the Evolutionary and Developmental Priority of Semantically Thick Words". Philosophy of Science 77, no. 5 (2010): 1096-1108.

$\mathrm{Xu}$, Fei, and Joshua B. Tenenbaum. "Word Learning as Bayesian Inference". Psychological Review 114, no. 2 (2007): 245-272.

Millikan, Ruth G. "A common structure for concepts of individuals, stuffs, and real kinds: More Mama, more milk, and more mouse”. Behavioral and Brain Sciences 21, no. 1 (1998): 55-65.

Jackson-Maldonado, Donna, Donna Thal, Virginia Marchman, Elizabeth Bates, and Vera GutirrezClellen. "Early lexical development in Spanish-speaking infants and toddlers". Journal of child language 20, no. 03 (1993): 523-549.

Nelson, Katherine. "Structure and strategy in learning to talk". Monographs of the society for research in child development 1-2, no. 149 (1973): 1-135.

Sandhofer, Catherine, and Linda B. Smith. "Learning adjectives in the real world: How learning nouns impedes learning adjectives. Language Learning and Development". Language Learning and Development 3, no. 3 (2007): 233-267.

Gärdenfors, Peter. "From Sensations to Concepts: a Proposal for Two Learning Processes". Review of Philosophy and Psychology (2018): 1-24.

Marr, David. Vision: A Computational Investigation into the Human Representation and Processing of Visual Information. 19-38. New York: Freeman, 1982.

Gasser, Michael, and Linda B. Smith. "Learning nouns and adjectives: A connectionist account. Language and cognitive processes". Language and cognitive processes 13, numbers 2-3 (1998): 269-306. 
O’Callaghan, Casey. “Auditory Perception”. In The Stanford Encyclopedia of Philosophy, Winter 2016, ed. by Edward N. Zalta. Metaphysics Research Lab, Stanford University, 2016.

Brössel, Peter. "Rational relations between perception and belief: the case of color". Review of Philosophy and Psychology (2017): 721-744.

Colombo, Matteo. "Bayesian cognitive science, predictive brains, and the nativism debate". Synthese (2017): 1-22.

Brössel, Peter, and Nina Poth. "Learning words and acquiring concepts". Manuscript.

Poth, Nina. "A Bayesian Approach Towards Concept Learning and an Answer to the Complex First Paradox”. MA thesis, Ruhr-University Bochum, 2016.

Gärdenfors, Peter. Conceptual Spaces: The Geometry of Thought. Cambridge, Massachusetts: MIT Press, 2000.

Shepard, Roger N. "Toward a universal law of generalization for psychological science”. Science 237, no. 4820 (1987): 1317-1323.

Rosch, Eleanor, and Carolyn B. Mervis. "Family resemblances: studies in the internal structure of categories". Cognitive Psychology 7, no. 4 (1975): 573-605.

Rosch, Eleanor. "Cognitive representations of semantic categories." Journal of experimental psychology: General 104, no. 3 (1975): 192-233.

Horton, Marjorie S., and Ellen M. Markman. "Developmental differences in the acquisition of basic and superordinate categories”. Child development 51, no. 3 (1980): 708-719.

Mervis, Carolyn B., and Maria A. Crisafi. "Order of acquisition of subordinate-, basic-, and superordinate-level categories". Child Development 53, no. 1 (1982): 258-266.

Smith, Linda B., Susan S. Jones, Barbara Landau, Lisa Gershkoff-Stowe, and Larissa Samuelson. "Object name learning provides on-the-job training for attention". Psychological Science 13, no. 1 (2002): 13-19. 
Jones, Susan S., Linda B. Smith, and Barbara Landau. "Object properties and knowledge in early lexical learning". Child development 62, no. 3 (1991): 499-516.

Landau, Barbara, Linda B. Smith, and Susan S. Jones. "The importance of shape in early lexical learning". Cognitive development 3, no. 3 (1988): 299-321.

$\mathrm{Xu}$, Fei, and Joshua B. Tenenbaum. "Word learning as Bayesian inference: Evidence from preschoolers". In Proceedings of the twenty-seventh annual conference of the cognitive science society, vol. 23. 2005.

Smith, Linda B., and Larissa Samuelson. "An attentional learning account of the shape bias: Reply to Cimpian and Markman (2005) and Booth, Waxman, and Huang (2005)." Developmental Psychology 42, no. 6 (2006): 1339-1343.

Kemp, Charles, Amy Perfors, and Joshua B. Tenenbaum. "Learning overhypotheses with hierarchical Bayesian models". Developmental science 10, no. 3 (2007): 307-321. 